\title{
Some results concerning certain periodic continued fractions
}

\author{
by \\ Kell Cheng and Hugh Williams (Calgary)
}

1. Introduction. One of the most important invariants of an order of an algebraic number field is the regulator of that order. It has been known for over two hundred years that the fundamental unit of such an order can be determined from the periodic continued fraction expansion of the generator of the order. In this case the regulator is simply the logarithm of the fundamental unit.

For most real quadratic orders the regulator tends to be rather large, but in certain, unusual and infrequent cases it is small, ensuring that the ideal class number will be large. Such orders are of very great interest to number theorists because the corresponding ideal class groups often have exotic structures. The search for such orders is a very old problem in number theory (see [3]). This is done in the context of determining families of values of non-square $D$ for which the period length $\ell$ of the regular continued fraction of $\sqrt{D}$ is small. Since the regulator of the order $\mathcal{O}(=\mathbb{Z}+\mathbb{Z} \sqrt{D})$ can be easily bounded above by $\ell(\log 2 \sqrt{D})$, this means that the regulator of $\mathcal{O}$ will be small.

In this paper, we study classes of integer-valued quadratic polynomials $D(X)$ for which the regular continued fraction expansion of $\sqrt{D(X)}$ exhibits a predictable pattern as $X$ varies. For instance, see [2], [5], [10] and [14].

The most important results concerning the regular continued fraction expansion of $\sqrt{D(X)}$ were established by Schinzel [11], [12]. He showed that if $D(X)=a^{2} X^{2}+b X+c$, where $a, b, c \in \mathbb{Z}, X \in \mathbb{N}$ and $a \neq 0$, then $\sqrt{D(X)}$ has bounded period length if and only if the discriminant $b^{2}-4 a c$ divides $4 \operatorname{gcd}\left(2 a^{2}, b\right)^{2}$. Also, Louboutin [7] and Farhane [4] later gave lower bounds for the case where the divisibility condition fails.

2000 Mathematics Subject Classification: Primary 11A55; Secondary 11R27, 11Y11, $11 \mathrm{Y} 65$.

Key words and phrases: continued fractions, period length, fundamental units.

Research partially supported by NSERC of Canada. 
Schinzel elegantly established this result without computing the actual continued fraction expansion of $\sqrt{D(X)}$. This prompted van der Poorten and Williams [9] to study the continued fraction expansion of $\sqrt{D(X)}$ that has bounded period length. They first established that by examining the cases of even $X$ and odd $X$ separately, there is no loss of generality in considering only quadratics of the form

$$
D(X)=A^{2} X^{2}+2 B X+C,
$$

where $A>0, B$ and $C$ are integers. Under this consideration, Schinzel's divisibility condition becomes

$$
B^{2}-A^{2} C \mid 4 \operatorname{gcd}\left(A^{2}, B\right)^{2},
$$

the Schinzel condition. Assuming $\operatorname{gcd}\left(A^{2}, 2 B, C\right)$ to be squarefree, van der Poorten and Williams obtained the exact expansion of $\sqrt{D(X)}$ for sufficiently large $X$. Moreover, the period length of the expansion of $\sqrt{D(X)}$ in this case is constant and is independent of $X$. Besides van der Poorten and Williams, Mollin [8] also studied the actual continued fraction expansion of $\sqrt{D(X)}$ from a different perspective.

In this article, we drop the condition that $\operatorname{gcd}\left(A^{2}, 2 B, C\right)$ be squarefree but maintain the divisibility condition $B^{2}-A^{2} C \mid 4 \operatorname{gcd}\left(A^{2}, B\right)^{2}$, and seek the continued fraction expansion of $\sqrt{D(X)}$ for sufficiently large $X$. We take a constructive approach in explicitly computing the expansion of $\sqrt{D(X)}$. As a consequence of this approach, we find the period length of the expansion to be constant with respect to the congruence classes of $X$ modulo a certain integer whose value depends only on $A, B, C$. Also, we are able to establish an upper bound for the period length of the expansion of $\sqrt{D(X)}$ and produce a closed form for the fundamental unit (and consequently the regulator) of the real quadratic order $[1, \sqrt{D(X)}]=\mathbb{Z}+\mathbb{Z} \sqrt{D(X)}$ when $X$ is sufficiently large.

We begin with some preliminary remarks about continued fractions. If $\theta$ is any real number, we can find the regular continued fraction (RCF) expansion of $\theta$, denoted as

$$
\theta=\left(a_{0}, a_{1}, \ldots, a_{n-1}, \theta_{n}\right),
$$

by putting $\theta_{0}=\theta, a_{0}=\left\lfloor\theta_{0}\right\rfloor$ and defining the complete quotient as $\theta_{i+1}=$ $1 /\left(\theta_{i}-a_{i}\right)$ and the partial quotient as $a_{i+1}=\left\lfloor\theta_{i+1}\right\rfloor$ for $i=0,1, \ldots$

If $\theta$ is an irrational, we know that the RCF expansion of $\theta$ contains an infinite number of partial quotients and that the ordered set of partial quotients is unique to $\theta$. If $\theta$ is a rational, we know that the RCF expansion is finite. That is,

$$
\theta=\left(a_{0}, a_{1}, \ldots, a_{m}\right)
$$


where $a_{0} \in \mathbb{Z}, a_{i} \in \mathbb{N}(i=1, \ldots, m)$. If $\theta \neq 1$, there is some ambiguity about $m$, however, as $a_{m}=\left(a_{m}-1,1\right)\left(a_{m}>1\right)$ and $\left(a_{m-1}, 1\right)=a_{m-1}+1$. This ambiguity can be easily resolved if we specify the parity of $m$ or insist that the last partial quotient be greater than 1 .

If $\theta$ is a real quadratic irrational, we may assume with no loss of generality that $\theta=(P+\sqrt{D}) / Q$, where $D, P, Q \in \mathbb{Z}, D>0, \sqrt{D} \notin \mathbb{Q}$ and $Q \mid D-P^{2}$. In this case, we can put $P_{0}=P, Q_{0}=Q$ and $\theta_{i}=\left(P_{i}+\sqrt{D}\right) / Q_{i}$. The values of $P_{i}$ and $Q_{i}$ can be computed recursively by

$$
P_{i+1}=a_{i} Q_{i}-P_{i}, \quad Q_{i+1}=\left(D-P_{i+1}^{2}\right) / Q_{i}
$$

for $i=0,1, \ldots$. It is well known that the RCF expansion of a quadratic irrational ultimately becomes periodic. We signal the periodic part of the continued fraction expansion by putting a bar over it. For example,

$$
\sqrt{7}=(2,1,1,1,4,1,1,1,4,1,1,1,4, \ldots)=(2, \overline{1,1,1,4}) .
$$

We define the convergents $C_{i}(i=0,1, \ldots)$ of $\left(a_{0}, a_{1}, \ldots, a_{n-1}, \theta_{n}\right)$ to be the values of the continued fraction $\left(a_{0}, a_{1}, \ldots, a_{i}\right)(i=0,1, \ldots)$. These can be easily computed by defining $A_{-2}=0, A_{-1}=1, B_{-2}=1, B_{-1}=0$, and computing

$$
A_{j+1}=a_{j+1} A_{j}+A_{j-1}, \quad B_{j+1}=a_{j+1} B_{j}+B_{j-1}
$$

recursively for $j=-1,0,1, \ldots$ Then $C_{i}=A_{i} / B_{i}$. Also, it is well known that $A_{i} B_{i-1}-B_{i} A_{i-1}=(-1)^{i-1}$.

Notice that in our definition of a RCF expansion of $\theta$, we have all partial quotients $a_{i}>0$, where $i \geq 1$. However, this condition can be relaxed in a formal continued fraction. In this case we simply write

$$
\theta=\left\langle a_{0}, a_{1}, \ldots, a_{n-1}, \theta_{n}\right\rangle,
$$

where $a_{i} \in \mathbb{Z}(i=0,1, \ldots, n-1)$. Here we have $\theta_{i+1}=1 /\left(\theta_{i}-a_{i}\right)$ as before. The convergents are defined similarly to the case of the RCF and

$$
\theta_{n}=-\frac{B_{n-2} \theta-A_{n-2}}{B_{n-1} \theta-A_{n-1}}
$$

just as in the RCF case. Here and in what follows, the bracket notation \langle\rangle stands for the formal continued fraction while the parenthesis notation ( ) continues to represent the regular continued fraction.

2. Preliminary results. In this section we present two general results needed for our work. The first result deals with $\lfloor\sqrt{D(X)}\rfloor$ and the second result deals with the embedding of a sequence $s_{0}, s_{1}, \ldots, s_{m-1}$ in a continued fraction. 
For any quadratic $D(X)=A^{2} X^{2}+2 B X+C$, not necessarily satisfying the Schinzel condition, we may write

$$
D(X)=\left(\frac{A^{2} X+B}{A}\right)^{2}-\frac{\Delta}{A^{2}}
$$

where $\Delta=B^{2}-A^{2} C$. A simple verification will secure the following result.

Theorem 2.1. Write $B=A q+r$ with $0 \leq r<A$. Then

$$
\lfloor\sqrt{D(X)}\rfloor= \begin{cases}A X+q-1 & \text { if } \Delta>0, r=0 \text { and } X>\frac{\Delta}{2 A^{3}}-\frac{2 B-A}{2 A^{2}}, \\ A X+q & \text { if } \Delta>0, r>0 \text { and } X>\frac{\Delta}{2 A^{2} r}-\frac{2 B-r}{2 A^{2}}, \\ A X+q & \text { if } \Delta<0, r=0 \text { and } X>\frac{-\Delta}{2 A^{3}}-\frac{2 B+A}{2 A^{2}} \\ A X+q & \text { if } \Delta<0, r>0 \text { and } X>\frac{-\Delta}{2 A^{2}(A-r)}-\frac{2 B+(A-r)}{2 A^{2}} .\end{cases}
$$

Let $s_{0}, s_{1}, \ldots, s_{m-1}$ be any sequence of integers and $\theta=(P+\sqrt{D}) / Q$ be a quadratic irrational. Further, let $\theta^{*}=(\mathbf{P}+\sqrt{D}) / \mathbf{Q}$ be defined by

$$
\theta=\left\langle s_{0}, s_{1}, \ldots, s_{m-1}, \theta^{*}\right\rangle .
$$

As indicated earlier, we have

$$
\theta^{*}=-\frac{B_{m-2} \theta-A_{m-2}}{B_{m-1} \theta-A_{m-1}}
$$

After some simple algebraic manipulations, we obtain

$$
\begin{aligned}
& \mathbf{P}=(-1)^{m}\left(B_{m-1} B_{m-2} Q^{\prime}-A_{m-1} A_{m-2} Q+\left(A_{m-1} B_{m-2}+A_{m-2} B_{m-1}\right) P\right), \\
& \mathbf{Q}=(-1)^{m}\left(A_{m-1}^{2} Q-B_{m-1}^{2} Q^{\prime}-2 A_{m-1} B_{m-1} P\right),
\end{aligned}
$$

where $Q^{\prime}=\left(D-P^{2}\right) / Q \in \mathbb{Z}$.

Let $d \in \mathbb{N}$ and put $F=d A_{m-1}$ and $E=d B_{m-1}$. Since $\operatorname{gcd}\left(A_{m-1}, B_{m-1}\right)$ $=1$, we get $d=\operatorname{gcd}(E, F)$ and

$$
\begin{aligned}
& \mathbf{P}=\frac{(-1)^{m}}{d}\left(B_{m-2} E Q^{\prime}-A_{m-2} F Q+\left(B_{m-2} F+A_{m-2} E\right) P\right), \\
& \mathbf{Q}=\frac{(-1)^{m}}{d^{2}}\left(F^{2} Q-E^{2} Q^{\prime}-2 E F P\right) .
\end{aligned}
$$

Theorem 2.2. Let $\theta=\left(P_{0}+\sqrt{D}\right) / Q_{0}$ be a quadratic irrational and put

$$
a_{0}=\left\lfloor\frac{P_{0}+\sqrt{D}}{Q_{0}}\right\rfloor, \quad P_{1}=a_{0} Q_{0}-P_{0}, \quad Q_{1}=\frac{D-P_{1}^{2}}{Q_{0}} .
$$

Let $L^{2} D=M^{2}-N$, where $L, M \in \mathbb{N}$ and $N \in \mathbb{Z}$, and put $F=L Q_{0}$, $E=M-L P_{1} \neq 0$ and $d=\operatorname{gcd}(E, F)$. Let the regular continued fraction expansion of $F / E$ be given by

$$
\left(s_{0}, s_{1}, \ldots, s_{m-1}\right),
$$


where $m$ is chosen to be odd if $N>0$ and even if $N<0$. If $(\mathbf{P}+\sqrt{D}) / \mathbf{Q}$ $(\mathbf{P}, \mathbf{Q} \in \mathbb{Z})$ is defined by

$$
\theta=\left\langle a_{0}, s_{0}, s_{1}, \ldots, s_{m-1},(\mathbf{P}+\sqrt{D}) / \mathbf{Q}\right\rangle,
$$

then

$$
\mathbf{P}=\frac{M}{L}-\frac{H|N|}{d L}, \quad \mathbf{Q}=\frac{|N| Q_{0}}{d^{2}},
$$

where $H=1$ if $F=E$, and $H(E / d) \equiv(-1)^{m-1} \bmod F / d(0 \leq H<F / d)$ otherwise.

Proof. Let $\left(P_{1}+\sqrt{D}\right) / Q_{1}$ be the first complete quotient of the regular continued fraction expansion of $\left(P_{0}+\sqrt{D}\right) / Q_{0}$. Then the values of $\mathbf{P}$ and $\mathbf{Q}$ are defined by

$$
\frac{P_{1}+\sqrt{D}}{Q_{1}}=\left\langle s_{0}, s_{1}, \ldots, s_{m-1}, \frac{\mathbf{P}+\sqrt{D}}{\mathbf{Q}}\right\rangle .
$$

If we substitute $Q_{1}$ for $Q, P_{1}$ for $P, Q_{0}=\left(D-P_{1}^{2}\right) / Q_{1}$ for $Q^{\prime}, M-L P_{1}$ for $E$ and $L Q_{0}$ for $F$ in (3), then we get

$$
\begin{aligned}
\mathbf{Q} & =\frac{(-1)^{m}}{d^{2}} Q_{0}\left(L^{2}\left(D-P_{1}^{2}\right)-\left(M-L P_{1}\right)^{2}-2\left(L P_{1}\right)\left(M-L P_{1}\right)\right) \\
& =\frac{(-1)^{m}}{d^{2}} Q_{0}\left(L^{2} D-\left(L P_{1}+M-L P_{1}\right)^{2}\right)=\frac{|N| Q_{0}}{d^{2}} .
\end{aligned}
$$

Similarly, by (2),

$$
\begin{aligned}
L \mathbf{P} & =\frac{(-1)^{m}}{d}\left(B_{m-2} L M Q_{0}-A_{m-2} L\left(L Q_{0}\right) Q_{1}+A_{m-2}\left(M-L P_{1}\right) L P_{1}\right) \\
& =\frac{(-1)^{m}}{d}\left(B_{m-2} L M Q_{0}+A_{m-2} L M P d_{1}-A_{m-2} M^{2}+A_{m-2} N\right) .
\end{aligned}
$$

Since $L Q_{0}=A_{m-1} d$ and $M-L P_{1}=B_{m-1} d$, we have

$$
B_{m-2} L Q_{0}-A_{m-2}\left(M-L P_{1}\right)=\left(A_{m-1} B_{m-2}-A_{m-2} B_{m-1}\right) d=(-1)^{m} d .
$$

Thus,

$$
\mathbf{P}=\frac{(-1)^{m}(-1)^{m} d M}{d L}+\frac{(-1)^{m} A_{m-2} N}{d L}=\frac{M}{L}-\frac{A_{m-2}|N|}{d L} .
$$

Put $H=A_{m-2}$. It follows that $H=1$ when $F=E$, and $H(E / d) \equiv$ $(-1)^{m-1} \bmod F / d(0 \leq H<F)$ otherwise. In either case, we may write

$$
\mathbf{P}=\frac{M}{L}-\frac{H|N|}{d L}
$$

The above result will play a pivotal role in our approach. We will construct the regular continued fraction expansion of $\sqrt{D(X)}$ by combining segments of the expansion. These segments are found by applying the above theorem. Note that the above result gives a segment for some continued 
fraction, which may not be regular. Thus, we need a criterion to determine when it is regular.

TheOREM 2.3. Let $\theta$ be an irrational number and suppose that

$$
\theta=\left\langle a_{0}, a_{1}, \ldots, a_{n-1}, \theta_{n}^{*}\right\rangle
$$

where $a_{1}, \ldots, a_{n-1} \in \mathbb{N}$. If $\theta^{*}>1$, then

$$
\theta=\left(a_{0}, a_{1}, \ldots, a_{n-1}, \theta_{n}\right),
$$

where $\theta_{n}=\theta_{n}^{*}$.

Proof. Since $\theta^{*}>1$, the partial quotients in the RCF expansion of $\theta^{*}$ are all in $\mathbb{N}$. Thus, the result of the theorem follows easily by the uniqueness of the RCF expansion of $\theta$.

Remark 2.1. If $F>E$, i.e. $s_{0} \in \mathbb{N}$, and $(\mathbf{P}+\sqrt{D}) / \mathbf{Q}>1$ in Theorem 2.2, then by Theorem 2.3, the RCF expansion of $\theta$ is given by

$$
\left(a_{0}, s_{0}, s_{1}, \ldots, s_{m-1},(\mathbf{P}+\sqrt{D}) / \mathbf{Q}\right) .
$$

3. Main results. Before we get to the main results, we need to introduce some notation. Assume that

$$
\Delta=B^{2}-A^{2} C \mid 4 \operatorname{gcd}\left(A^{2}, B\right)^{2} \quad \text { and } \quad|\Delta|=\Delta_{1} \Delta_{2}^{2} \Delta_{4}^{4},
$$

where $\Delta_{1}$ and $\Delta_{2}$ are squarefree. It follows that

$$
\Delta_{1} \Delta_{2} \Delta_{4}^{2}\left|2 A^{2}, \quad \Delta_{1} \Delta_{2} \Delta_{4}^{2}\right| 2 B
$$

Also, since $\left|B^{2}-A^{2} C\right|=\Delta_{1} \Delta_{2}^{2} \Delta_{4}^{4}$, we see that if $G=\operatorname{gcd}(A, B)$, then

$$
G=\operatorname{gcd}\left(A, \Delta_{2} \Delta_{4}^{2}\right) \text {. }
$$

We define

$$
A^{\prime}=A / G, \quad \Delta^{\prime}=\Delta_{2} \Delta_{4}^{2} / G .
$$

Note that $\operatorname{gcd}\left(A^{\prime}, \Delta^{\prime}\right)=1$, and by (4), we have $\Delta_{1} \Delta^{\prime} \mid 2 A$. Also, since $\Delta_{1} \Delta^{\prime} \mid 2 B$, we have $\Delta_{1} \Delta^{\prime} \mid 2 G$. If $A \mid B$, then $A=G$ and $A^{\prime}=1$. Define $\sigma=\Delta /|\Delta|=\operatorname{sgn}(\Delta)$, and

$$
\eta= \begin{cases}1 & \text { if } A \mid B \text { and } \sigma=1 \\ 0 & \text { otherwise }\end{cases}
$$

For integers $a \geq r \geq 0$, define an ordered set

$$
\text { (8) } \mathcal{S}(a, r)= \begin{cases}\emptyset & \text { if } r=0, \\ \left\{s_{0}, s_{1}, \ldots, s_{m-1}\right\} & \text { otherwise, } \\ & \text { where } a / r=\left(s_{0}, s_{1}, \ldots, s_{m-1}\right) \\ & \text { with }(-1)^{m-1}=\sigma .\end{cases}
$$

Finally, we define $\varepsilon_{i} \in\{0,1\}, \varepsilon_{i} \equiv i \bmod 2$. Note that $\varepsilon_{i+1}=1-\varepsilon_{i}$. 
As we mentioned earlier, our result on the continued fraction expansion of $\sqrt{D(X)}$ requires $X$ to be sufficiently large. Henceforth, we say $X$ is sufficiently large if $X$ is a non-negative integer such that

$$
X>\frac{|\Delta|}{A G^{2}}+\frac{1}{2 A}-\frac{B}{A^{2}} \text { and } X>1+\frac{1}{A}-\frac{B}{A^{2}} .
$$

It is easy to see that when the first inequality is satisfied, $X$ is greater than the lower bounds in Theorem 2.1. The second inequality is needed for reasons that will become clear later. We let $X \equiv K \bmod \Delta^{\prime}$, where $0 \leq K<\Delta^{\prime}$, and write $B=A q+r$, where $0 \leq r<A$.

TheOREM 3.1 (The regular continued fraction expansion of $\sqrt{D(X)}$ ). Suppose that $D(X)=A^{2} X^{2}+2 B X+C$ satisfies the Schinzel condition, where $X$ is sufficiently large, i.e., (9) holds. Write $X=W \Delta^{\prime}+K$ for some $W \geq 0$. Put $d_{0}=\Delta^{\prime}$ and $r_{0}=(r+A \eta) / G$ and inductively define the following.

For $i \geq 0$, define

$$
\mathcal{S}_{i}=\mathcal{S}\left(A^{\prime} \Delta^{\prime} / d_{i}, r_{i}\right), \quad d_{i+1}=\operatorname{gcd}\left(\Delta^{\prime} / d_{i}, r_{i}\right),
$$

where the parity of $\left|\mathcal{S}_{i}\right|$ is even if $\sigma=-1$ and odd if $\sigma=1$. Put $g_{i+1}=0$ or $g_{i+1}=d_{i}$ according as $r_{i}=0$ or $r_{i}=A^{\prime} \Delta^{\prime} / d_{i}$. If $r_{i} \not \equiv 0 \bmod A^{\prime} \Delta^{\prime} / d_{i}$, then choose $g_{i+1} \in \mathbb{Z}$ so that

$$
\frac{g_{i+1}}{d_{i}} \frac{r_{i}}{d_{i+1}} \equiv \sigma \bmod \frac{A^{\prime} \Delta^{\prime}}{d_{i} d_{i+1}} \quad \text { and } \quad 0<g_{i+1}<\frac{A^{\prime} \Delta^{\prime}}{d_{i+1}} .
$$

Also,

$q_{i+1}(X)=\frac{2 A W d_{i+1}^{2}}{\Delta_{1}^{\varepsilon_{i+1}} \Delta}+\left\lfloor\frac{2 A^{2} K+2 B-\Delta_{1}^{\varepsilon_{i+1}} g_{i+1}\left(A / A^{\prime}\right)\left(\Delta^{\prime} / d_{i+1}\right)-A \eta-r}{A \Delta_{1}^{\varepsilon_{i+1}}\left(\Delta^{\prime} / d_{i+1}\right)^{2}}\right\rfloor$.

Compute $r_{i+1}$ such that

$$
r_{i+1} \equiv \frac{d_{i+1}\left(2 A^{2} K+2 B\right)}{\Delta_{1}^{\varepsilon_{i+1}} \Delta_{2} \Delta_{4}^{2}}-g_{i+1} \bmod \frac{A^{\prime} \Delta^{\prime}}{d_{i+1}},
$$

where $0 \leq r_{i+1}<A^{\prime} \Delta^{\prime} / d_{i+1}$ when $\sigma=-1$ and $0<r_{i+1} \leq A^{\prime} \Delta^{\prime} / d_{i+1}$ when $\sigma=1$.

Then the regular continued fraction expansion of $\sqrt{D(X)}$ is given by

$$
\left(A X+q-\eta, \overline{\mathcal{S}_{0}, q_{1}(X), \mathcal{S}_{1}, q_{2}(X), \ldots, \mathcal{S}_{\kappa-1}, q_{\kappa}(X)}\right),
$$

where $\eta$ is defined by (7) and $\kappa$ is the least natural number such that

$$
d_{\kappa}=\Delta^{\prime} \quad \text { and } \quad \Delta_{1}^{\varepsilon_{\kappa}}=1 .
$$

Proof. We prove the result by induction on the subscript $i$. We do this by establishing formulas for $\mathbf{P}_{i}$ and $\mathbf{Q}_{i}$ such that

$$
\sqrt{D(X)}=\left(A X+q-\eta, \mathcal{S}_{0},\left(\mathbf{P}_{1}+\sqrt{D(X)}\right) / \mathbf{Q}_{1}\right)
$$


and

$$
\left(\mathbf{P}_{i}+\sqrt{D(X)}\right) / \mathbf{Q}_{i}=\left(q_{i}(X), \mathcal{S}_{i},\left(\mathbf{P}_{i+1}+\sqrt{D(X)}\right) / \mathbf{Q}_{i+1}\right)
$$

for $i \geq 1$.

When $X$ is sufficiently large, it follows from Theorem 2.1 that

$$
\lfloor\sqrt{D(X)}\rfloor=A X+q-\eta \text {. }
$$

Initial step: Put $\mathbf{P}_{0}=0$ and $\mathbf{Q}_{0}=1$. Then

$$
\sqrt{D(X)}=\left(\mathbf{P}_{0}+\sqrt{D(X)}\right) / \mathbf{Q}_{0}, \quad P_{1}=a_{0} \mathbf{Q}_{0}-\mathbf{P}_{0}=A X+q-\eta .
$$

In view of Theorem 2.2, we put $L=A, M=A X^{2}+B$ and $N=\Delta$, so that

$$
E=M-L P^{\prime}=r+A \eta, \quad F=L \mathbf{Q}_{0}=A .
$$

Let $d=\operatorname{gcd}(F, E)=\operatorname{gcd}(A, r+A \eta)$. Then $d=\operatorname{gcd}(A, r)=G$ by (5). Put $r_{0}=(r+A \eta) / G$. Since $A^{\prime}=A / G$, we have $d_{1}=\left(A^{\prime}, r_{0}\right)=1$. By the definition of $\eta$, it is clear that $0 \leq E \leq F$, i.e., $0 \leq r_{0} \leq A^{\prime}$. If $E=0$, we must have $r=0=\eta$, i.e., $A \mid B$ and $\sigma=-1$. If $E=F$, then $r=0$ and $\eta=1$, i.e., $A \mid B$ and $\sigma=1$. Also, if $r>0$, then $\eta=0$ and $0<E<F$.

CASE (1). Suppose that $E=0$, i.e., $r_{0}=0$ and $\sigma=-1$. In this case, we cannot apply Theorem 2.2. We put $\mathcal{S}_{0}=\emptyset$ and compute

$$
\mathbf{P}_{1}=P^{\prime}=A X+\frac{B}{A}, \quad \mathbf{Q}_{1}=\frac{D(X)-\mathbf{P}_{1}^{2}}{\mathbf{Q}_{0}}=\frac{-\Delta}{A^{2}}=\Delta_{1}\left(\frac{\Delta^{\prime}}{A^{\prime}}\right)^{2} .
$$

CASe (2). Assume that $E \neq 0$, i.e., $0<r_{0} \leq A^{\prime}$. Let the RCF expansion of $A /(r+A \eta)$ be given by $\left(s_{0}, s_{1}, \ldots, s_{m-1}\right)$, where $(-1)^{m-1}=\sigma$. By Theorem 2.2, we get integers

$$
\mathbf{P}_{1}=\frac{A^{2} X+B}{A}-\frac{H|\Delta|}{A d}, \quad \mathbf{Q}_{1}=\frac{|\Delta|}{d^{2}},
$$

where $H=1$ if $F=E$, and $H \cdot(r+A \eta) / d \equiv \sigma \bmod A / d(0 \leq H<A / d)$ otherwise.

Note that

$$
\frac{H|\Delta|}{A d}=\Delta_{1} \frac{H \Delta^{\prime 2}}{A^{\prime}} \text { and } \quad \frac{|\Delta|}{d^{2}}=\Delta_{1}\left(\frac{\Delta^{\prime}}{d_{1}}\right)^{2} .
$$

Set

$$
g_{1}=H \Delta^{\prime}, \quad d_{0}=\Delta^{\prime} .
$$

If $r_{0}=A^{\prime}$, then $g_{1}=d_{0}$; otherwise, $g_{1}$ satisfies

$$
\frac{g_{1} r_{0}}{d_{0} d_{1}} \equiv \sigma \bmod \frac{A^{\prime} \Delta^{\prime}}{d_{0} d_{1}} \quad \text { and } \quad 0<g_{1}<\frac{A^{\prime} \Delta^{\prime}}{d_{1}} .
$$

Also,

$$
\mathbf{P}_{1}=\frac{A^{2} X+B}{A}-\Delta_{1} \frac{g_{1}}{A^{\prime}} \frac{\Delta^{\prime}}{d_{1}}, \quad \mathbf{Q}_{1}=\Delta_{1}\left(\frac{\Delta^{\prime}}{d_{1}}\right)^{2}
$$


Put $\mathcal{S}_{0}=\mathcal{S}\left(A^{\prime}, r_{0}\right)$. We now need to establish that

$$
\left(A X+q-\eta, \mathcal{S}_{0},\left(\mathbf{P}_{1}+\sqrt{D(X)}\right) / \mathbf{Q}_{1}\right)
$$

is indeed the regular continued fraction expansion of $\sqrt{D(X)}$. By Remark 2.1, since we know that $F>E$, we need $\left(\mathbf{P}_{1}+\sqrt{D(X)}\right) / \mathbf{Q}_{1}>1$. This can be easily verified under the assumption that $X>|\Delta| /\left(A G^{2}\right)-$ $(2 B-A) /\left(2 A^{2}\right)$.

Now, regardless of Case (1) or (2), we have $d_{0}=\Delta^{\prime}, \mathcal{S}_{0}=\mathcal{S}\left(\Delta^{\prime} / d_{0}, r_{0}\right)$ and $d_{1}=\operatorname{gcd}\left(A^{\prime} \Delta^{\prime} / d_{0}, r_{0}\right)=1$. When $r_{0} \equiv 0 \bmod A^{\prime}$, put $g_{1}=0$ or $g_{1}=d_{0}$ according as $r_{0}=0$ or $r_{0}=A^{\prime}$; otherwise, choose $g_{1}$ according to (14). Also,

$$
\mathbf{P}_{1}=\frac{A^{2} X+B}{A}-\Delta_{1} \frac{g_{1}}{A^{\prime}} \frac{\Delta^{\prime}}{d_{1}}, \quad \mathbf{Q}_{1}=\Delta_{1}\left(\frac{\Delta^{\prime}}{d_{1}}\right)^{2} .
$$

Since $X=W \Delta^{\prime}+K$, where $0 \leq K<\Delta^{\prime}$, it is not difficult to deduce that

$$
\begin{aligned}
q_{1}(X) & =\left\lfloor\frac{\mathbf{P}_{1}+\sqrt{D(X)}}{\mathbf{Q}_{1}}\right\rfloor \\
& =\frac{2 A W d_{1}^{2}}{\Delta_{1} \Delta^{\prime}}+\left\lfloor\frac{2 A^{2} K+2 B-\Delta_{1} g_{1}\left(A / A^{\prime}\right)\left(\Delta^{\prime} / d_{1}\right)-A \eta-r}{A \Delta_{1}\left(\Delta^{\prime} / d_{1}\right)^{2}}\right\rfloor .
\end{aligned}
$$

Inductive step. For some $i \in \mathbb{N}$, suppose that $d_{i}>0, d_{i} \mid \Delta^{\prime}$ and $g_{i} \geq 0$ are integers and $\left(\mathbf{P}_{i}+\sqrt{D(X)}\right) / \mathbf{Q}_{i}$ is a complete quotient of the RCF expansion of $\sqrt{D(X)}$, where

$$
\mathbf{P}_{i}=A X+\frac{B}{A}-\Delta_{1}^{\varepsilon_{i}} \frac{g_{i}}{A^{\prime}} \frac{\Delta^{\prime}}{d_{i}}, \quad \mathbf{Q}_{i}=\Delta_{1}^{\varepsilon_{i}}\left(\frac{\Delta^{\prime}}{d_{i}}\right)^{2}
$$

are integers. Let $q_{i}(X)=\left\lfloor\left(\mathbf{P}_{i}+\sqrt{D(X)}\right) / \mathbf{Q}_{i}\right\rfloor, \mathbf{P}^{\prime}=q_{i}(X) \mathbf{Q}_{i}-\mathbf{P}_{i}$ and

$$
\mathbf{R}_{i}=\left(\mathbf{P}_{i}+\lfloor\sqrt{D(X)}\rfloor\right)-q_{i}(X) \mathbf{Q}_{i}
$$

where $0 \leq \mathbf{R}_{i}<\mathbf{Q}_{i}$. Since $0 \leq r / A+\eta \leq 1$, we have $0 \leq \mathbf{R}_{i}+r / A+\eta \leq \mathbf{Q}_{i}$, that is,

$$
0 \leq A \mathbf{R}_{i}+r+A \eta \leq A \mathbf{Q}_{i} .
$$

Note that if $r=0$ and $\sigma=-1$, then $\eta=0$ and the above inequalities become $0 \leq A \mathbf{R}_{i}+A<A \mathbf{Q}_{i}$. If $r=0$ and $\sigma=1$, then $\eta=1$ and we get $0<A \mathbf{R}_{i} \leq A \mathbf{Q}_{i}$. Also, if $r>0$, then $0<A \mathbf{R}_{i}+r<A \mathbf{Q}_{i}$.

In Theorem 2.2, we put

$$
F=A \mathbf{Q}_{i}, \quad E=A^{2} X+B-A \mathbf{P}^{\prime}=A \mathbf{R}_{i}+r+A \eta .
$$

Hence, $0 \leq E \leq F$ by (18). If $E=0$, i.e., $A \mathbf{R}_{i}+r+A \eta=0$, then $\mathbf{R}_{i}=0=$ $r=\eta$, and hence, $\sigma=-1$. If $E=F$, then $\mathbf{R}_{i}+r / A+\eta=\mathbf{Q}_{i}$. It follows that $r=0$ and $\mathbf{R}_{i}+\eta=\mathbf{Q}_{i}$. Since $\mathbf{R}_{i}<\mathbf{Q}_{i}$, we have $\eta=1$, which implies $\sigma=1$. 
We now note that

$$
A \mathbf{R}_{i}+r+A \eta \equiv 2 A^{2} X+2 B-A \Delta_{1}^{\varepsilon_{i}} \frac{g_{i}}{A^{\prime}} \frac{\Delta^{\prime}}{d_{i}} \bmod A \Delta_{1}^{\varepsilon_{i}}\left(\frac{\Delta^{\prime}}{d_{i}}\right)^{2} .
$$

By the assumption that $X \equiv K \bmod \Delta^{\prime}$, we have

$$
2 A^{2} X \equiv 2 A^{2} K \bmod A \Delta_{1}^{\varepsilon_{i}}\left(\frac{\Delta^{\prime}}{d_{i}}\right)^{2} .
$$

Hence,

$$
A \mathbf{R}_{i}+r+A \eta \equiv 2 A^{2} K+2 B-A \Delta_{1}^{\varepsilon_{i}} \frac{g_{i}}{A^{\prime}} \frac{\Delta^{\prime}}{d_{i}} \bmod A \Delta_{1}^{\varepsilon_{i}}\left(\frac{\Delta^{\prime}}{d_{i}}\right)^{2} .
$$

Notice that $\left(\Delta_{1}^{\varepsilon_{i}} \Delta_{2} \Delta_{4}^{2} / d_{i}\right)$ divides every term on the right of the above congruence as well as the modulus, so it must also divide $A \mathbf{R}_{i}+r+A \eta$. Put

$$
r_{i}=\frac{A \mathbf{R}_{i}+r+A \eta}{\Delta_{1}^{\varepsilon_{i}} \Delta_{2} \Delta_{4}^{2} / d_{i}} .
$$

Since $0 \leq E \leq F$, we get $0 \leq r_{i} \leq A^{\prime} \Delta^{\prime} / d_{i}$. Also,

$$
r_{i} \equiv \frac{d_{i}\left(2 A^{2} K+2 B\right)}{\Delta_{1}^{\varepsilon_{i}} \Delta_{2} \Delta_{4}^{2}}-g_{i} \bmod \frac{A^{\prime} \Delta^{\prime}}{d_{i}}
$$

We note that $r_{i}=0$ if and only if $E=0$. Similarly, $r_{i}=A^{\prime} \Delta^{\prime} / d_{i}$ if and only if $E=F$.

CASE (1). If $r_{i}=0$, then $r=0$ and $A^{\prime}=1$ and we compute

$$
\mathbf{P}_{i+1}=q_{i}(X) \mathbf{Q}_{i}-\mathbf{P}_{i}=A X+\frac{B}{A}, \quad \mathbf{Q}_{i+1}=\frac{D(X)-\mathbf{P}_{i+1}^{2}}{\mathbf{Q}_{i}}=\Delta_{1}^{\varepsilon_{i+1}}\left(\frac{d_{i}}{A^{\prime}}\right)^{2} .
$$

Put $\mathcal{S}_{i}=\mathcal{S}\left(A^{\prime} \Delta^{\prime} / d_{i}, r_{i}\right)=\emptyset, d_{i+1}=\Delta^{\prime} / d_{i}$ and $g_{i+1}=0$, and write

$$
\mathbf{P}_{i+1}=A X+\frac{B}{A}-\Delta_{1}^{\varepsilon_{i+1}} \frac{g_{i+1}}{A^{\prime}} \frac{\Delta^{\prime}}{d_{i+1}}, \quad \mathbf{Q}_{i+1}=\Delta_{1}^{\varepsilon_{i+1}}\left(\frac{\Delta^{\prime}}{d_{i+1}}\right)^{2} .
$$

CASE (2). Suppose that $r_{i}>0$, i.e., $0<r_{i} \leq A^{\prime} \Delta^{\prime} / d_{i}$. Let the RCF expansion of $F / E=\left(A^{\prime} \Delta^{\prime} / d_{i}\right) / r_{i}$ be given by $\left(s_{0}, s_{1}, \ldots, s_{m-1}\right)$, where $m$ is chosen so that $(-1)^{m-1}=\sigma$. Put

$$
\mathcal{S}_{i}=\mathcal{S}\left(\frac{A^{\prime} \Delta^{\prime}}{d_{i}}, r_{i}\right)
$$

Let $d=\operatorname{gcd}(E, F)$ and $d_{i+1}=\operatorname{gcd}\left(A^{\prime} \Delta^{\prime} / d_{i}, r_{i}\right)$. Then $d=\left(\Delta_{1}^{\varepsilon_{i}} \Delta_{2} \Delta_{4}^{2} / d_{i}\right) d_{i+1}$.

If $E=F$, put $H=1$; otherwise, choose $H$ such that

$$
H \frac{E}{d} \equiv \sigma \bmod \frac{F}{d} \quad \text { and } \quad 0 \leq H<\frac{F}{d} .
$$

Then, by Theorem 2.2, we get

$$
\mathbf{P}_{i+1}=A X+\frac{B}{A}-\frac{H|\Delta|}{A d} \quad \text { and } \quad \mathbf{Q}_{i+1}=\frac{|\Delta| \mathbf{Q}_{i}}{d^{2}} .
$$


Since $E / d \equiv r_{i} / d_{i+1} \bmod F / d$ and $F / d=\left(A^{\prime} \Delta^{\prime}\right) /\left(d_{i} d_{i+1}\right)$, (22) can be written as

$$
H \frac{r_{i}}{d_{i+1}} \equiv \sigma \bmod \frac{A^{\prime} \Delta^{\prime}}{d_{i} d_{i+1}} \quad \text { and } \quad 0 \leq H<\frac{A^{\prime} \Delta^{\prime}}{d_{i} d_{i+1}}
$$

Also,

$$
\frac{H|\Delta|}{d A}=\frac{d_{i} H \Delta_{1}^{\varepsilon_{i+1}} \Delta^{\prime}}{A^{\prime} d_{i+1}} \text { and } \frac{|\Delta| \mathbf{Q}_{i}}{d^{2}}=\Delta_{1}^{\varepsilon_{i+1}}\left(\frac{\Delta^{\prime}}{d_{i+1}}\right)^{2} .
$$

Put $g_{i+1}=d_{i} H$; it follows that $g_{i+1}=d_{i}$ if $r_{i}=A^{\prime} \Delta^{\prime} / d_{i}$, or $g_{i+1}$ satisfies

$$
\frac{g_{i+1} r_{i}}{d_{i} d_{i+1}} \equiv \sigma \bmod \frac{A^{\prime} \Delta^{\prime}}{d_{i} d_{i+1}} \quad \text { and } \quad 0<g_{i+1}<\frac{A^{\prime} \Delta^{\prime}}{d_{i+1}} .
$$

Moreover,

$$
\mathbf{P}_{i+1}=A X+\frac{B}{A}-\Delta_{1}^{\varepsilon_{i+1}} \frac{g_{i+1}}{A^{\prime}} \frac{\Delta^{\prime}}{d_{i+1}} \quad \text { and } \quad \mathbf{Q}_{i+1}=\Delta_{1}^{\varepsilon_{i+1}}\left(\frac{\Delta^{\prime}}{d_{i+1}}\right)^{2} .
$$

It is a simple matter to verify $\left(\mathbf{P}_{i+1}+\sqrt{D(X)}\right) / \mathbf{Q}_{i+1}>1$ using the assumption that $X$ satisfies the first inequality in (9). Hence, by Remark 2.1, the expansion of $\left(\mathbf{P}_{i}+\sqrt{D(X)}\right) / \mathbf{Q}_{i}$ is given by

$$
\left(q_{i}(X), \mathcal{S}_{i}, \frac{\mathbf{P}_{i+1}+\sqrt{D(X)}}{\mathbf{Q}_{i+1}}\right) .
$$

Also, it is easy to check that $q_{i+1}(X)=\left\lfloor\left(\mathbf{P}_{i+1}+\sqrt{D(X)}\right) / \mathbf{Q}_{i+1}\right\rfloor$ is given by

$$
\frac{2 A W d_{i+1}^{2}}{\Delta_{1}^{\varepsilon_{i+1}} \Delta^{\prime}}+\left\lfloor\frac{2 A^{2} K+2 B-\Delta_{1}^{\varepsilon_{i+1}} g_{i+1}\left(A / A^{\prime}\right)\left(\Delta^{\prime} / d_{i+1}\right)-A \eta-r}{A \Delta_{1}^{\varepsilon_{i+1}}\left(\Delta^{\prime} / d_{i+1}\right)^{2}}\right\rfloor .
$$

By induction (on the subscript $i$ ), the regular continued fraction expansion of $\sqrt{D(X)}$ is given by

$$
\left(A X+q-\eta, \mathcal{S}_{0}, q_{1}(X), \mathcal{S}_{1}, q_{2}(X), \ldots\right) .
$$

Since $d_{0}=\Delta^{\prime}$ and $\mathbf{Q}_{i}=\Delta_{1}^{\varepsilon_{i}}\left(\Delta^{\prime} / d_{i}\right)^{2} \in \mathbb{N}$ for all $i \in \mathbb{N}$, it follows that $d_{i} \mid \Delta^{\prime}$ for all $i \geq 0$. Moreover, since $\operatorname{gcd}\left(A^{\prime}, \Delta^{\prime}\right)=1$, we get $d_{i+1}=\operatorname{gcd}\left(\Delta^{\prime} / d_{i}, r_{i}\right)$ for all $i \geq 0$. Also, by the definition of $g_{i+1}$, we have $d_{i} \mid g_{i+1}$ for all $i \geq 0$.

Since $\sqrt{D(X)}$ is a quadratic irrational, its continued fraction expansion is periodic. The end of the period is signaled by $Q_{\ell}=1$ for some minimal $\ell \in \mathbb{N}$ and the corresponding partial quotient takes the form $2 A X+2 q-2 \eta$, twice the initial partial quotient. Since the partial quotients in the sequences $\mathcal{S}_{i}$ are all less than $A^{\prime} \Delta^{\prime}$, by the second inequality of (9) and the fact that $2 G \geq \Delta_{1} \Delta^{\prime}$, the only partial quotient that can be as large as $2 A X+2 q-2 \eta$ must be $q_{\kappa}(X)$ for some $\kappa$. Hence, if $\ell$ is the period length, then $Q_{\ell}=1$ and 
$P_{\ell}=A X+q-\eta ;$ since

$$
Q_{\ell}=\mathbf{Q}_{\kappa}=\Delta_{1}^{\varepsilon_{\kappa}}\left(\frac{\Delta^{\prime}}{d_{\kappa}}\right)^{2} \quad \text { and } \quad P_{\ell}=\mathbf{P}_{\kappa}=A X+\frac{B}{A}-\Delta_{1}^{\varepsilon_{\kappa}} \frac{g_{\kappa}}{A^{\prime}} \frac{\Delta^{\prime}}{d_{\kappa}},
$$

we must have $\Delta_{1}^{\varepsilon_{\kappa}}=1$ and $d_{\kappa}=\Delta^{\prime}$. In other words, our computation for the regular continued fraction expansion of $\sqrt{D(X)}$ is complete when we get the minimal $\kappa>0$ such that $\Delta_{1}^{\varepsilon_{\kappa}}=1$ and $d_{\kappa}=\Delta^{\prime}$.

REMARKS 3.1. (1) It is clear that $\Delta_{1}>1$ implies that $\kappa$ must be even.

(2) We see that $\mathcal{S}_{0}$ is independent of the residue classes of $X$ modulo $\Delta^{\prime}$ and $\mathcal{S}_{i}$ with $i \geq 1$ depends on the residue classes. In particular, if $r=0$, i.e., $A \mid B$, then $\mathcal{S}_{0}=\emptyset$ or $\{1\}$ according as $\sigma=-1$ or 1 . Also, when $r_{i} \equiv$ $0 \bmod A^{\prime} \Delta^{\prime} / d_{i}$ for $i \geq 1, \mathcal{S}_{i}=\emptyset$ or $\{1\}$ according as $\sigma=-1$ or 1 . Further, if $r>0$, then $r_{i}>0$ for $i \geq 0$.

ExAmple 3.1. Consider $D(X)=119^{2} X^{2}+2(2205) X+343$. We first look at $X \equiv 1 \bmod 7$, where $X \geq 1$, and list $q_{i}(X), \mathcal{S}_{i}, g_{i}, d_{i}$ and $r_{i}$ :

$$
\begin{aligned}
& q_{0}(1)=137, \quad \mathcal{S}_{0}=\{1,1,8\}, \quad d_{0}=\Delta^{\prime}=7, \quad r_{0}=9 \text {, } \\
& q_{1}(1)=2, \quad \mathcal{S}_{1}=\{1,2,4,1,1,1,2\}, \quad d_{1}=1, \quad g_{1}=14, \quad r_{1}=82, \\
& q_{2}(1)=5, \quad \mathcal{S}_{2}=\{4,3,1\}, \quad d_{2}=1, \quad g_{2}=45, \quad r_{2}=28 \text {, } \\
& q_{3}(1)=136, \quad \mathcal{S}_{3}=\{1,3,4\}, \quad d_{3}=7, \quad g_{3}=13, \quad r_{3}=13, \\
& q_{4}(1)=5, \quad \mathcal{S}_{4}=\{2,1,1,1,4,2,1\}, \quad d_{4}=1, \quad g_{4}=28, \quad r_{4}=45, \\
& q_{5}(1)=2, \quad \mathcal{S}_{5}=\{8,1,1\}, \quad d_{5}=1, \quad g_{5}=82, \quad r_{5}=14 \text {, } \\
& q_{6}(1)=274, \quad \mathcal{S}_{6}=\{1,1,8\}, \quad d_{6}=\Delta^{\prime}=7, \quad r_{6}=9 .
\end{aligned}
$$

Since $\varepsilon_{6}=0$ by the definition of $\varepsilon_{i}$, we have $\Delta_{1}^{\varepsilon_{6}}=1$. Also, since $d_{6}=\Delta^{\prime}$, the computation of $\mathcal{S}_{i}$ of the continued fraction expansion of $\sqrt{D(1)}$ is complete and $\kappa=6$. Hence, when $X=7 W+1$ for $W \geq 0$, we have

$$
\begin{array}{r}
\sqrt{D(7 W+1)}=\left(833 W+137, \overline{1,1,8, q_{1}(W), 1,2,4,1,1,1,2, q_{2}(W), 4,3,1, q_{3}(W)},\right. \\
\left.\overline{1,3,4, q_{4}(W), 2,1,1,1,4,2,1, q_{5}(W), 8,1,1,2(833 W+137)}\right),
\end{array}
$$

where $q_{1}(W)=q_{5}(W)=17 W+2, q_{2}(W)=q_{4}(W)=34 W+5$ and $q_{3}(W)=$ $833 W+136$.

Similarly, when $X=7 W+2$ and $W \geq 0$,

$$
\sqrt{D(7 W+2)}=(833 W+256, \overline{1,1,8,17 W+5,8,1,1,2(833 W+256)}) .
$$

When $X=7 W+3$ and $W \geq 0$,

$$
\sqrt{D(7 W+3)}=\left(833 W+375, \overline{1,1,8, q_{1}(W), 1,1,4,1,10, q_{2}(W), 4,3,1, q_{3}(W)},\right.
$$


where $q_{1}(W)=q_{5}(W)=17 W+7, q_{2}(W)=q_{4}(W)=34 W+15$ and $q_{3}(W)=833 W+374$.

When $X=7 W+4$ and $W \geq 0$, the continued fraction expansion of $\sqrt{D(7 W+4)}$ is given by

$\left(833 W+494, \overline{1,1,8, q_{1}(W), 1,38,1,1,1, q_{2}(W), 1,1,11,2,2, q_{3}(W), 1,2,4,1,1,1,2, q_{4}(W)}\right.$,

$\overline{1,4,5,1,3, q_{5}(W), 1,4,1,18,1, q_{6}(W), 4,3,1, q_{7}(W), 1,3,4, q_{8}(W), 1,18,1,4,1, q_{9}(W)}$,

$\overline{3,1,5,4,1, q_{10}(W), 2,1,1,1,1,4,2,1, q_{11}(W), 2,2,11,1,1, q_{12}(W), 1,1,1,38,1, q_{13}(W)}$,

$\overline{8,1,1,2(833 W+494)})$,

where $q_{1}(W)=q_{3}(W)=q_{5}(W)=q_{9}(W)=q_{11}(W)=q_{13}(W)=17 W+9$, $q_{2}(W)=q_{4}(W)=q_{6}(W)=q_{8}(W)=q_{10}(W)=q_{12}(W)=34 W+19$ and $q_{7}(W)=833 W+493$.

When $X=7 W+5$ and $W \geq 0$,

$\sqrt{D(7 W+5)}=\left(833 W+613, \overline{1,1,8, q_{1}(W), 2,2,11,1,1, q_{2}(W)}\right.$,

$\left.\overline{2,2,11,1,1, q_{3}(W), 8,1,1,2(833 W+613)}\right)$,

where $q_{1}(W)=q_{3}(W)=17 W+12$ and $q_{2}(W)=34 W+24$.

When $X=7 W+6$ and $W \geq 0$,

$\sqrt{D(7 W+6)}=\left(833 W+732, \overline{1,1,8, q_{1}(W), 1,4,1,18,1, q_{2}(W)}\right.$,

$$
\left.\overline{1,18,1,4,1, q_{3}(W), 8,1,1,2(833 W+732)}\right),
$$

where $q_{1}(W)=q_{3}(W)=17 W+14$ and $q_{2}(W)=34 W+28$.

When $X=7 W$ and $W \geq 1$, the continued fraction expansion of $\sqrt{D(7 W)}$ is given by

$\left(833 W+18, \overline{1,1,8, q_{1}(W), 3,1,5,4,1, q_{2}(W), 1,18,1,4,1, q_{3}(W), 1,1,4,1,10, q_{4}(W)}\right.$,

$\overline{1,1,3,8,1, q_{5}(W), 2,2,11,1,1, q_{6}(W), 4,3,1, q_{7}(W), 1,3,4, q_{8}(W), 1,1,11,2,2, q_{9}(W)}$,

$\overline{1,38,1,1,1, q_{10}(W), 10,1,4,1,1, q_{11}(W), 1,4,1,18,1, q_{12}(W), 1,4,5,1,3, q_{13}(W)}$,

$$
\overline{8,1,1,2(833 W+18)}) \text {, }
$$

where $q_{1}(W)=q_{13}(W)=17 W, q_{2}(W)=q_{12}(W)=34 W-1, q_{3}(W)=$ $q_{5}(W)=q_{9}(W)=q_{11}(W)=17 W-1, q_{4}(W)=q_{6}(W)=q_{8}(W)=$ $q_{10}(W)=34 W$ and $q_{7}(W)=833 W+17$.

4. Upper bound for $\operatorname{lp}(\sqrt{D(X)})$. In this section, we give a simple presentation concerning an explicit upper bound for the period length of the RCF expansion of $\sqrt{D(X)}$, denoted by $\operatorname{lp}(\sqrt{D(X)})$. The details of the results here can be found in Cheng [1, Chapter 5]. 
By Theorem 3.1, we know that the calculation of the RCF expansion of $\sqrt{D(X)}$ ends when $d_{\kappa}=\Delta^{\prime}$ and $\Delta_{1}^{\varepsilon_{\kappa}}=1$ for some minimal $\kappa$. Hence, if we write

$$
\left|\mathcal{S}_{i}\right|=\left|\mathcal{S}\left(\frac{A^{\prime} \Delta^{\prime}}{d_{i}}, r_{i}\right)\right|=\left|\mathcal{S}\left(A^{\prime} \Delta, d_{i} r_{i}\right)\right|
$$

as the cardinality of the set $\mathcal{S}_{i}$, then

$$
\operatorname{lp}(\sqrt{D(X)})=\sum_{i=0}^{\kappa-1}\left(1+\left|\mathcal{S}_{i}\right|\right)
$$

Recall that $\left|\mathcal{S}_{i}\right|$ is the length of the RCF expansion of $A^{\prime} \Delta^{\prime} / d_{i} r_{i}$ when $r_{i}>0$, and $\left|\mathcal{S}_{i}\right|=0$ when $r_{i}=0$. We henceforth consider $r_{i}>0$.

It is well known (see, for example, Knuth [6, p. 343]) that the maximum length for the RCF expansion of $a / r$ is at $\operatorname{most}\left\lfloor\log _{\varphi}(\sqrt{5} \cdot a)\right\rfloor-1$. Hence, $\left|\mathcal{S}_{i}\right| \leq\left\lfloor\log _{\varphi}\left(\sqrt{5} \cdot A^{\prime} \Delta^{\prime}\right)\right\rfloor-1$ and

$$
\operatorname{lp}(\sqrt{D(X)}) \leq \sum_{i=0}^{\kappa-1}\left(1+\left\lfloor\log _{\varphi}\left(\sqrt{5} \cdot A^{\prime} \Delta^{\prime}\right)\right\rfloor-1\right)=\kappa \cdot\left\lfloor\log _{\varphi}\left(\sqrt{5} \cdot A^{\prime} \Delta^{\prime}\right)\right\rfloor
$$

It remains to determine an upper bound on the value of $\kappa$. Define

$$
T=\frac{2 A^{2} K+2 B}{\Delta_{2} \Delta_{4}^{2}}, \quad P=\frac{T^{2}}{\Delta_{1}}-2 \sigma .
$$

Let $\alpha$ and $\beta$ be roots of $x^{2}-P x+1=0$. Then $\alpha+\beta=P$ and $\alpha \beta=1$. Define

$$
U_{n}=\frac{\alpha^{n}-\beta^{n}}{\alpha-\beta}
$$

and let $\omega(m)$ be the rank of apparition of $m$ in the Lucas function $U_{n}$.

It was shown in [1, Chapter 5] that $\kappa \mid 2 \omega\left(\Delta^{\prime}\right)$ and that $\omega(m) \leq m$ if $m$ is odd and $\omega(m) \leq(3 / 2) m$ if $m$ is even. Hence, we have the following result.

THEOREM 4.1.

$$
\operatorname{lp}(\sqrt{D(X)}) \leq \begin{cases}3 \Delta^{\prime}\left\lfloor\log _{\varphi}\left(\sqrt{5} \cdot A^{\prime} \Delta^{\prime}\right)\right\rfloor & \text { if } \Delta^{\prime} \text { is even }, \\ 2 \Delta^{\prime}\left\lfloor\log _{\varphi}\left(\sqrt{5} \cdot A^{\prime} \Delta^{\prime}\right)\right\rfloor & \text { if } \Delta^{\prime} \text { is odd }\end{cases}
$$

where $\varphi=(1+\sqrt{5}) / 2$ and $\log _{\varphi}(x)$ is the logarithm of $x$ with base $\varphi$.

The result $\kappa \mid 2 \omega\left(\Delta^{\prime}\right)$ can be improved to

TheOREM 4.2. The value of $\kappa$ is either $2 \omega\left(\Delta^{\prime} / \delta\right)$ or $\omega\left(\Delta^{\prime}\right)$, where $\delta=$ $\operatorname{gcd}\left(\Delta^{\prime}, T / \Delta_{1}\right)$. The latter case can occur only if $\omega\left(\Delta^{\prime}\right)$ is odd and $\Delta_{1}=1$.

Example 4.1. Consider $D(X)=119^{2} X^{2}+2(833) X+245$. Then $A=119$, $B=833, C=245, \Delta=-2^{2} \cdot 7^{4} \cdot 17^{2}$ and $\sigma=-1$. Also, $\Delta_{1}=1, \Delta_{2}=34$, $\Delta_{4}=7$ and $\Delta^{\prime}=14$. As $K$ varies from 0 to 13 , we first compute $\kappa$ and then either $\omega\left(\Delta^{\prime}\right)$ or $\omega\left(\Delta^{\prime} / \delta\right)$ and arrive at Table 1 : 
Table 1. Values of $\kappa$ and $\omega\left(\Delta^{\prime} / \delta\right)$ of $\sqrt{119^{2} X^{2}+2(833) X+245}$

\begin{tabular}{c|cccccccccccccc}
\hline$K$ & 0 & 1 & 2 & 3 & 4 & 5 & 6 & 7 & 8 & 9 & 10 & 11 & 12 & 13 \\
\hline$\kappa$ & 24 & 8 & 6 & 8 & 24 & 6 & 6 & 8 & 24 & 2 & 24 & 8 & 6 & 6 \\
\hline$\omega\left(\Delta^{\prime} / \delta\right)$ & 12 & 4 & 3 & 4 & 12 & 6 & 3 & 4 & 12 & 1 & 12 & 4 & 3 & 3 \\
\hline
\end{tabular}

Remark 4.1. The results in [9] assume that $\operatorname{gcd}\left(A^{2}, 2 B, C\right)$ is squarefree. This assumption leads to $\Delta^{\prime} \mid 2$. It was shown in [1, Chapter 5] that $\kappa$ in this case is $1,2,3,4$ or 6 .

5. Fundamental unit of $[1, \sqrt{D(X)}]$. Stender $[13]$ studied the fundamental unit of the order $[1, \sqrt{D(X)}]$, where the radicand $D(X)$ is assumed to be squarefree for some sufficiently large integer $X$. In this section, we make use of Theorem 3.1 to get the fundamental unit of the real quadratic order $[1, \sqrt{D(X)}]$ for sufficiently large $X$.

It is well known that if $\varepsilon$ is the fundamental unit of $[1, \sqrt{D}]$ for some nonsquare natural number $D, \ell=\operatorname{lp}(\sqrt{D})$ and $\theta_{i}$ is the $i$ th complete quotient in the RCF expansion of $\sqrt{D}$, then

$$
\varepsilon=\prod_{i=1}^{\ell} \theta_{i}
$$

Lemma 5.1. Let $\theta(X)=(P+\sqrt{D(X)}) / Q$ be a complete quotient of $\sqrt{D(X)}, q(X)=\lfloor(P+\sqrt{D(X)}) / Q\rfloor$ and $E$ and $F$ as defined in Theorem 2.2 so that $F / E=\left(s_{0}, s_{1}, \ldots, s_{m-1}\right)$ and

$$
\frac{P+\sqrt{D(X)}}{Q}=\left(q(X), s_{0}, s_{1}, \ldots, s_{m-1}, \theta^{*}(X)\right),
$$

where the parity of $m$ is chosen so that $(-1)^{m-1}=\Delta /|\Delta|$. If $\theta_{j}(X)$ is the jth complete quotient of $(P+\sqrt{D(X)}) / Q$, then

$$
\prod_{j=1}^{m+1} \theta_{j}(X)=\frac{d\left(A^{2} X+B+A \sqrt{D(X)}\right)}{|\Delta|},
$$

where $d=\operatorname{gcd}(E, F)$.

Proof. Let $A_{j} / B_{j}$ be the $j$ th convergent of $\theta(X)$. Then by (1), we have

$$
\theta_{j}(X)=-\frac{B_{j-2} \theta(X)-A_{j-2}}{B_{j-1} \theta(X)-A_{j-1}}
$$


for $j \geq 0$. Thus,

$$
\prod_{j=1}^{m+1} \theta_{j}(X)=\frac{(-1)^{m+1}}{B_{m} \theta(X)-A_{m}}
$$

Since $A_{m} / B_{m}=\left(q(X), s_{0}, s_{1}, \ldots, s_{m-1}\right)$, we have

$$
\frac{A_{m}}{B_{m}}=q(X)+\frac{1}{\left(s_{0}, s_{1}, \ldots, s_{m-1}\right)}=q(X)+\frac{E}{F}=\frac{q(X) F+E}{F} .
$$

Recall from Theorem 2.2 that $E=A^{2} X+B-A P^{\prime}$ and $F=A Q$, where $P^{\prime}=q(X) Q-P$. If $d=\operatorname{gcd}(E, F)$, then $d=\operatorname{gcd}(q(X) F+E, F)$. Hence,

$$
d A_{m}=q(X) F+E=q(X) A Q+A^{2} X+B-A P^{\prime}, \quad d B_{m}=A Q .
$$

So,

$$
B_{m} \theta(X)-A_{m}=\frac{1}{d}\left(A Q \theta(X)-q(X) A Q-A^{2} X-B+A P^{\prime}\right) .
$$

Since

$$
\begin{aligned}
& A Q \theta(X)-q(X) A Q+A P^{\prime} \\
& \quad=A Q\left(\frac{P+\sqrt{D(X)}}{Q}\right)-q(X) A Q+A(q(X) Q-P)=A \sqrt{D(X)},
\end{aligned}
$$

we have

$$
B_{m} \theta(X)-A_{m}=-\frac{A^{2} X+B-A \sqrt{D(X)}}{d} .
$$

Hence,

$$
\prod_{j=1}^{m+1} \theta_{j}(X)=\frac{(-1)^{m+1}}{B_{m} \theta(X)-A_{m}}=\frac{d\left(A^{2} X+B+A \sqrt{D(X)}\right)}{|\Delta|} .
$$

TheOREM 5.1. The fundamental unit $\varepsilon$ of $[1, \sqrt{D(X)}]$ for $X$ satisfying (9) is given by

$$
\varepsilon=|\Delta|^{\kappa / 2}\left(\frac{A^{2} X+B+A \sqrt{D(X)}}{|\Delta|}\right)^{\kappa}=\left(\frac{A^{2} X+B+A \sqrt{D(X)}}{\sqrt{|\Delta|}}\right)^{\kappa} .
$$

Moreover, the norm of $\varepsilon$ is $\sigma^{\kappa}$. By Remarks 3.1, $\kappa$ is even when $\Delta_{1}>1$, so $|\Delta|^{\kappa / 2} \in \mathbb{N}$.

Proof. By Theorem 3.1,

$$
\sqrt{D(X)}=\left(q_{0}(X), \overline{\mathcal{S}_{0}, q_{1}(X), \mathcal{S}_{1}, \ldots, \mathcal{S}_{\kappa-1}, q_{\kappa}(X)}\right) .
$$

For $i \geq 0$, let $\theta_{i}(X)=\left(q_{i}(X), \mathcal{S}_{i}, \theta_{i+1}(X)\right), \theta_{j}^{\prime}(X)$ be the $j$ th complete quotient of $\theta_{i}(X)$ and

$$
\vartheta_{i}(X)=\prod_{j=1}^{1+\left|\mathcal{S}_{i}\right|} \theta_{j}^{\prime}(X)
$$


Further, let $\theta_{k}(X)$ be the $k$ th complete quotient of $\sqrt{D(X)}$ and $\ell=$ $\sum_{i=0}^{\kappa-1}\left(1+\left|\mathcal{S}_{i}\right|\right)$. Then by the remark at the beginning of this section, the fundamental unit $\varepsilon$ of $[1, \sqrt{D(X)}]$ is given by

$$
\varepsilon=\prod_{i=1}^{\ell} \theta_{i}(X)=\prod_{i=0}^{\kappa-1} \vartheta_{i}(X)
$$

By Lemma 5.1, we have

$$
\vartheta_{i}(X)=\frac{\delta_{i+1}\left(A^{2} X+B+A \sqrt{D(X)}\right)}{|\Delta|},
$$

where $\delta_{1}=G d_{1}$ and $\delta_{i}=\left(\Delta_{1}^{\varepsilon_{i-1}} \Delta_{2} \Delta_{4}^{2} / d_{i-1}\right) d_{i}$ for $i \geq 2$. Thus,

$$
\begin{aligned}
\varepsilon & =\prod_{i=0}^{\kappa-1} \vartheta_{i}=\prod_{i=0}^{\kappa-1} \frac{\delta_{i+1}\left(A^{2} X+B+A \sqrt{D(X)}\right)}{|\Delta|} \\
& =\left(\frac{A^{2} X+B+A \sqrt{D(X)}}{|\Delta|}\right)^{\kappa} \prod_{i=1}^{\kappa} \delta_{i} .
\end{aligned}
$$

Now,

$$
\prod_{i=1}^{\kappa} \delta_{i}=G d_{1} \cdot \frac{\Delta_{1}^{\varepsilon_{1}} \Delta_{2} \Delta_{4}^{2}}{d_{1}} d_{2} \cdot \frac{\Delta_{1}^{\varepsilon_{2}} \Delta_{2} \Delta_{4}^{2}}{d_{2}} d_{3} \ldots \frac{\Delta_{1}^{\varepsilon_{\kappa-1}} \Delta_{2} \Delta_{4}^{2}}{d_{\kappa-1}} d_{\kappa} .
$$

Since $\varepsilon_{i}$ is either 0 or 1 according as $i$ is even or odd, and since $\kappa$ is even if $\Delta_{1}>1$ by Remarks 3.1, we may write

$$
\prod_{i=1}^{\kappa} \delta_{i}=G \Delta_{1}^{\kappa / 2}\left(\Delta_{2} \Delta_{4}^{2}\right)^{\kappa-1} d_{\kappa}
$$

Since $d_{\kappa}=\Delta^{\prime}$ by Theorem 3.1, we have $G d_{\kappa}=\Delta_{2} \Delta_{4}^{2}$. Thus,

$$
\prod_{i=1}^{\kappa} \delta_{i}=|\Delta|^{\kappa / 2} \text { and } \varepsilon=|\Delta|^{\kappa / 2}\left(\frac{A^{2} X+B+A \sqrt{D(X)}}{|\Delta|}\right)^{\kappa} .
$$

Now, the norm of $\varepsilon$ is

$$
\frac{|\Delta|^{\kappa}}{|\Delta|^{2 \kappa}} \cdot \mathcal{N}\left(A^{2} X+B+A \sqrt{D(X)}\right)^{\kappa}=\frac{|\Delta|^{\kappa}}{|\Delta|^{2 \kappa}} \Delta^{\kappa}=\frac{\Delta^{\kappa}}{|\Delta|^{\kappa}}=\sigma^{\kappa} \text {. }
$$

Corollary 5.1. If $\Delta_{1}>1$, then the norm of $\varepsilon$ is 1 .

\section{References}

[1] K. H. F. Cheng, Some results concerning periodic continued fractions, Ph.D. dissertation, Univ. of Calgary, 2003.

[2] G. Degert, Über die Bestimmung der Grundeinheit gewisser reell-quadratischer Zahlkörper, Abh. Math. Sem. Univ. Hamburg 22 (1958), 92-97. 
[3] L. E. Dickson, History of the Theory of Numbers, Vol. II, Chelsea, New York, 1952.

[4] A. Farhane, Minoration de la période du développement de $\sqrt{a^{2} n^{2}+b n+c}$ en fraction continue, Acta Arith. 67 (1994), 63-67.

[5] H. Hasse, Über mehrklassige, aber eingeschlechtige reell-quadratische Zahlkörper, Elem. Math. 20 (1965), 49-59.

[6] D. E. Knuth, The Art of Computer Programming, Vol. II: Seminumerical Algorithms, 2nd ed., Addison-Wesley, Reading, MA, 1981.

[7] S. Louboutin, Une version effective d'un théorème de A. Schinzel sur les longueurs des périodes de certains développements en fractions continues, C. R. Acad. Sci. Paris Sér. I Math. 308 (1989), 511-513.

[8] R. A. Mollin, Polynomials of Pellian type and continued fractions, Serdica Math. J. 27 (2001), 317-342.

[9] A. J. van der Poorten and H. C. Williams, On certain continued fraction expansions of fixed period length, Acta Arith. 89 (1999), 23-35.

[10] C. Richaud, Sur la résolution des équations $x^{2}-A y^{2}= \pm 1$, Atti Accad. Pontif. Nuovi Lincei 19 (1865), 177-182.

[11] A. Schinzel, On some problems of the arithmetical theory of continued fractions, Acta Arith. 6 (1961), 393-413.

[12] -, On some problems of the arithmetical theory of continued fractions $I I$, ibid. 7 (1962), 287-298.

[13] H.-J. Stender, Über die Grundeinheit der reell-quadratischen Zahlkörper $\mathbb{Q}\left(\sqrt{A^{2} N^{2}+B N+C}\right)$, J. Reine Angew. Math. 311/312 (1979), 302-306.

[14] M. A. Stern, Theorie der Kettenbrüche und ihre Anwendung. Siebentes Capitel. Anwendung der Kettenbrüche auf die höhere Arithmetik, ibid. 11 (1834), 326-341.

Mathematics and Statistics

University of Calgary

Calgary, Alberta

Canada, T2N $1 \mathrm{~N} 4$

E-mail: kellcheng@hotmail.com

williams@math.ucalgary.ca

Received on 18.2.2004

and in revised form on 3.11.2004 